\author{
Alina Dorota Jarząbek \\ ORCID: 0000-0001-5988-9906 \\ Warmia und Mazury Universität in Olsztyn, Olsztyn
}

DOI: $10.19195 / 0435-5865.143 .31$

\title{
(D)ACHL-Bilder. Zum Bildmaterial in polnischen DaF-Lehrwerken für das Gymnasium
}

\begin{abstract}
s
Der vorliegende Beitrag informiert über Ergebnisse einer Lehrwerkanalyse, in der Bilder der deutschsprachigen Länder (A, CH, L) in polnischen DaF-Lehrwerken für das Gymnasium eine zentrale Rolle spielen. Der theoretische Hintergrund wird dabei nur skizziert. Die Untersuchungsergebnisse lassen allgemein feststellen, dass polnische Gymnasialschüler ein ideales Bild der drei Länder präsentiert bekommen, das Postkarten oder Werbung auf touristischen Flyern ähnelt. Kontrastive, kritische oder provokative Bilder der Realität werden in den Lehrwerken völlig ausgelassen.
\end{abstract}

Schlüsselwörter: Bild, visuelles Material, DACH-Prinzip, Lehrwerkanalyse

\section{(D)ACHL Images. For visual material in Polish textbooks of German as a foreign language for lower secondary school}

The article presents the results of a textbook analysis of Polish textbooks of German. The analysis focuses on the images of the German-speaking countries (Austria, Switzerland, Liechtenstein). The theoretical background is only sketched. The study results lead to the general conclusion that Polish lower secondary school students are offered an ideal image of the three countries which is similar to that on postcards or advertising on tourist leaflets. Contrasting, critical and not beautiful pictures of reality are left out in the textbooks.

Keywords: image, visual material, a principle of DACH, analysis of textbook

Alina Jarząbek, Uniwersytet Warmińsko-Mazurski w Olsztynie, Katedra Filologii Germańskiej, ul. Obitza 1, pok.349, 10-725 Olsztyn, Polen, E-Mail: alina.jarzabek@uwm.edu.pl

Received: 24.07.2017, accepted: 18.05.2018 


\section{Einleitung}

Die Lehrwerkanalyse und -kritik polnischer DaF-Lehrwerke liegen seit einigen Jahren im Interessenbereich der Verfasserin (z.B. Jarząbek 2013a, 2013b, 2016a, 2016b, 2017). Im vorliegenden Beitrag wird das visuelle Material in den DaF-Lehrwerken für polnische Gymnasien gründlich untersucht. Schließlich sind Bilder wichtige Inhaltsträger in den Lehrwerken. Sie erregen die Aufmerksamkeit der Lernenden und eignen sich gut zur Vermittlung der Lerninhalte, insbesondere im Bereich der Landeskunde. Sie sind dabei für die Herstellung persönlicher und/ oder interkultureller Bezüge beim Bildbetrachter verantwortlich. Auf die Funktion der visuellen Medien im DaF-Unterricht machen z.B. Macaire/Hosch (1999), Schwerdtfeger (2001) und Rösler (2010) aufmerksam.

Der Begriff Bild ist im Deutschen mehrdeutig und wird zum Zweck dieses Beitrags und der durchgeführten Analyse als ,,mit künstlerischen Mitteln auf einer Fläche Dargestelltem, Wiedergegebenem, also einem Gemälde, einer Zeichnung o. Ä." sowie mit einer Fotografie und einer gedruckt wiedergegebenen bildlichen Darstellung gleichgesetzt. ${ }^{1}$

Das Bild als visuelles Medium wird zum Gegenstand der Bildwissenschaft (dazu z.B. Schöttler 2014) und der Rezeptionsforschung (dazu z.B. Bucher/Schumacher 2012). Bislang wurden Bilder nicht so oft zum Forschungsgegenstand im pädagogisch-didaktischen Kontext des DaF-/DaZ-Unterrichts, aber es gibt bereits interessante Befunde und Veröffentlichungen zu bewegten wie zu festen Bildern, beispielsweise von Maijala et al. (2016), Hieronimus (2014), Pešková (2014), Chudak (2013), Badstübner-Kizik (2006, 2007). ${ }^{2}$

Im Zentrum der vorliegenden Lehrwerkanalyse steht vor allem das ikonografische Material, das sich auf die deutschsprachigen Länder - Österreich, die Schweiz und Liechtenstein - bezieht. Der durchgeführten Untersuchung schließen sich einige didaktische Überlegungen an.

\section{Von ABCD-Thesen zum DACHL-Konzept und DACH-Prinzip}

Die politische Wende der 1990er-Jahre und die Wiedervereinigung Deutschlands haben eine früher kaum erwartete Änderung der landeskundlichen Inhalte in den DaF-Lehrwerken zur Folge gehabt. Bereits 1988 versammelte sich zum ersten Mal die Arbeitsgruppe der Deutschlehrerverbände aus den damaligen deutschsprachigen Ländern (A, BRD, DDR, CH) unter der Ägide des Internationalen

${ }^{1}$ www.duden.de/rechtschreibung/Bild (10.04.2016).

${ }^{2}$ Auf eine ausführlichere theoretische Fundierung des Bildbegriffes im Fremdsprachenunterricht und seiner weiteren Aspekte wird im Weiteren aus Platzgründen verzichtet. 
Deutschlehrerverbandes und verabschiedete die „ABCD-Thesen zur Rolle der Landeskunde im Deutschunterricht" (1990) (dazu neulich z.B. Sorger 2013). Die Thesen lieferten eine Neuorientierung der Landeskunde, die nun als Kombination von Sprachvermittlung und kulturellen Informationen definiert und gestaltet werden sollte. Sie führten somit auch zur Abkehr von einer faktischen, oft historisch ausgerichteten Landeskunde einzelner Länder, was zu Gunsten einer selbstständigen und aktiven Auseinandersetzung mit Kulturen des deutschsprachigen Raums geschah.

Das DACH-Prinzip und das DACH-Konzept können als eine modifizierte Fortsetzung der ABCD-Thesen betrachtet werden. Die beiden Termini werden in der Literatur oft synonym betrachtet (z.B. bei Demmig et al. 2013). Das DACH-Prinzip umfasst den inhaltlichen Anspruch, die grundsätzlichen Zielsetzungen und die theoretische Fundierung der Beschäftigung mit dem DACHL ${ }^{3}$ Raum und bezieht sich auf den 2008/2013 von der DACHL-Arbeitsgruppe verabschiedeten Grundsatz der Anerkennung und Berücksichtigung der sprachlichen und kulturellen Vielfalt des DACHL-Raums in DaF. ${ }^{4}$ Wie Demmig u.a. (2013) zeigen, braucht dieser Grundsatz allerdings noch eine theoretisch fundiertere Ausformulierung v. a. in der Unterrichtspraxis, Materialentwicklung, Lehrerbildung, aber auch im Rahmen sprachpolitischer und fachwissenschaftlicher Zusammenarbeit. Dabei geht es nicht um eine immer noch dominierende additive Betrachtung einzelner deutschsprachiger Länder, sondern um eine exemplarische Herangehensweise an die DACHL-landeskundlichen Inhalte, deren Umsetzung auf unterschiedliche Probleme stößt (dazu z.B. Maijala et al. 2016: 6).

\section{Forschungsgegenstand und -methode}

Wie bereits einleitend angemerkt wurde, sind Bilder der deutschsprachigen Länder in Regionallehrwerken für $\mathrm{DaF},{ }^{5}$ in dem Fall für polnische Gymnasien, von Interesse für den vorliegenden Beitrag, der die Analyse von fünf ausgewählten DaF-Lehrwerkreihen umfasst (s. Liste der analysierten Lehrwerke). Zwei davon, aha! Neu und Kompass neu gehören (nach internen Angaben der Lehrwerkverlage in Polen) zu den meist verkauften DaF-Lehrwerken für das Gymnasium und sind jeweils seit 2009 und 2012 auf dem Büchermarkt. Ein neues Lehrwerk ist hingegen Meine Deutschtour, erschienen 2015. Zwei weitere Titel, Magnet (ursprünglich publiziert von Loescher Editore, Turin 2007) und Beste Freunde (ursprünglich Hueber Verlag, Ismaning 2013) sind Produkte weder polnischer Verlage noch pol-

${ }^{3}$ Im Folgenden werden die Akronyme D, A, CH, L jeweils für Deutschland, Österreich, die Schweiz und Liechtenstein verwendet.

${ }^{4}$ www.idvnetz.org/dachl-online/das-dach-prinzip (Zugriff am 20.07.2016).

${ }^{5}$ Zur Entwicklung der regionalen Lehrwerke u.a. Breitung/Lattaro 2001: 1041 ff., Krumm 2010: $1221 \mathrm{f}$.). 
nischer Autoren und Autorinnen und wurden an die bildungsministerialen Zulassungskriterien für Lehrwerke zum Gebrauch an staatlichen Schulen in Polen angepasst. ${ }^{6}$ Die (subjektive) Auswahl dieses Forschungsmaterials beruht somit auf dem Prinzip einer Gegenüberstellung polnischer und nicht-polnischer Lehrwerke, ihrer zeitlichen Entstehung und ihrer Beliebtheit bei polnischen Deutschlehrkräften. Polnische Fremdsprachenlehrwerke sind nicht verpflichtet, das Sprachniveau auf dem jeweiligen Band zu vermerken. Es ist jedoch anzunehmen, dass die Lerner mit einer dreiteiligen DaF-Lehrwerkreihe das Sprachniveau A2 erreichen können.

Im vorliegenden Aufsatz wird der folgenden Forschungsfrage nachgegangen: Welches Bild von Österreich, der Schweiz und Liechtenstein wird in den DaF-Lehrwerken für polnische Gymnasien durch das darin enthaltene visuelle Material vermittelt ${ }^{7}$ ? Als geeignete Forschungsmethode bietet sich hierzu die Lehrwerkanalyse an (dazu z.B. Kast/Neuner 1994, Krumm/Ohms-Duschenko 2001, Schmidt 2001). Die Abbildungen werden quantitativ und qualitativ untersucht. Die qualitative Bildanalyse erfolgt durch eine Zuordnung der Bilder zu einer der folgenden inhaltlichen Kategorien:

- Alltagsleben

- Städte / Regionen / Geografie

- Politik / Soziales / Geschichte

- Sitten und Bräuche

- Persönlichkeiten

- Kultur / Architektur / Sehenswürdigkeiten

Bei der Identifizierung der Bildinhalte und der nachfolgenden Zuordnung des jeweiligen Bildes (eines Fotos, einer Zeichnung, einer Grafik etc.) zu einer Kategorie $^{8}$ spielen Bildunterschriften bzw. ein inhaltlicher Textbezug (bei Diagrammen und derartigen Grafiken) oder eine Aufgabenstellung, in der eindeutig ein Bezug auf A, $\mathrm{CH}$ oder L mitgeteilt wird, eine entscheidende Rolle. Das Bildmaterial, das keinen auffälligen Bezug zu A, CH oder L aufzeigt, wird aus der Aufzählung ausgeklammert.

${ }^{6}$ In Polen unterliegen alle Lehrwerke, die an öffentlichen Schulen gebraucht werden, einem Zulassungsverfahren anhand durch das Bildungsministerium festgelegter Kriterien. Die Lehrwerkprüfung erfolgt durch anerkannte Lehrwerkgutachter. Derzeit sind zwölf DaF-Lehrwerksreihen und ein Repetitorium zugelassen (Stand 17.07.2017). Mehr dazu unter www.podreczniki.men.gov.pl/ wykaz_dopuszczone_lista3.php.

${ }^{7}$ Zum Deutschland betreffenden Bildmaterial siehe Jarząbek (2016c).

${ }^{8}$ Pešková (2014) stellt ein methodologisches System der Kategorisierung und der Analyse von Typen, Funktionen und Inhalten von landeskundlichen Visualisierungen dar, das auf einer Entwicklung von Kategorien, Berechnung der Kodierer-Übereinstimmung und Durchführung einer Häufigkeits- und Kontingentanalyse beruht. Pešková hat die Analyse der Inhalte von landeskundlichen Visualisierungen unter zwei Aspekten, d.h. einem soziokulturellen (Subkategorien: Geographie, Geschichte, Staat und Politik, Kultur und Wissenschaft, Alltag, soziale Interaktion, nationale und soziale Identität) und einem geographischen (Subkategorien: Deutschland, Österreich, Schweiz, Liechtenstein und Luxemburg, mehrere deutschsprachige Länder, unbestimmt) durchgeführt. Dieses komplexe System zeichnet sich durch eine hohe Sensibilität und Ausführlichkeit aus, was auch oft den Mangel des Vorkommens mancher Subkategorien erweisen kann. 
Es wird dabei gleichzeitig angestrebt, Bilder in einem Bezug auf Textinhalte zu analysieren, denn eine solche Vorgehensweise ermöglicht und erleichtert eine Interpretation des Bildmaterials. Das sonstige visuelle Material, das keinen (D)ACHL-landeskundlichen Zusammenhang darstellt, wird bei der Bildevaluation ausgelassen.

\section{Forschungsergebnisse}

Im Weiteren werden die quantitativen und qualitativen Ergebnisse der Bildanalyse dargestellt. Tabelle 1 zeigt eine Zusammenstellung der ACHL-Bilder in den untersuchten DaF-Lehrwerken. ${ }^{9}$

Tab. 1. Bilderzahlen in den analysierten DaF-Lehrwerken

\begin{tabular}{|l|c|c|c|c|}
\hline \multicolumn{1}{|c|}{ Lehrwerk } & Bilder: A & Bilder: CH & Bilder: L & Gesamt \\
\hline aha! Neu (Band 1, 2, 3) & 5 & 1 & 0 & 6 \\
\hline Beste Freunde (Band 1, 2,3) & 21 & 18 & 5 & 44 \\
\hline Meine Deutschtour (Band 1,2,3) & 23 & 15 & 11 & 49 \\
\hline Kompass neu (Band 1, 2, 3) & 30 & 19 & 3 & 52 \\
\hline Magnet (Band 1, 2, 3, 4) & 18 & 11 & 4 & 33 \\
\hline Insgesamt [N] & 97 & 64 & 23 & 184 \\
\hline Prozent & 52,7 & 34,8 & 12,5 & 100 \\
\hline
\end{tabular}

In allen drei Bänden des Lehrwerks aha! Neu ist die Zahl dieser Bilder sehr spärlich [N=6]. Bei den vier anderen Lehrbüchern unterscheidet sich die gesamte Bilderzahl nicht so drastisch voneinander, wobei die meisten Abbildungen in der Lehrwerkreihe Kompass neu zu verzeichnen sind [ $\mathrm{N}=52]$. In diesen Lehrwerken finden die jungen Leser Bilder aus allen drei Ländern, obwohl die Bilderzahlen, die sich jeweils auf A, CH und L beziehen, ziemlich schwankend sind. Die Bilder Österreichs dominieren insgesamt in allen Lehrwerken [N=97], während die Abbildungen aus der Schweiz vergleichend um ein Drittel geringer darin auftreten [N=64]. Die Abbildungen Liechtensteins fehlen zwar in aha! Neu, aber zu begrüßen ist, dass alle anderen Lehrbücher dieses Land mit insgesamt 23 Nennungen präsentieren, bei Meine Deutschtour sogar mit 11.

In den folgenden Tabellen 2-7 werden sowohl quantitative als auch qualitative Zusammenstellungen der Bilder in den evaluierten DaF-Lehrwerken nach Kategorien geordnet präsentiert. Das jeweilige Bild wurde seinem Inhalt entspre-

${ }^{9}$ Die quantitative Analyse der Bilder zu Deutschland in denselben DaF-Lehrwerken hat insgesamt 257 Abbildungen ergeben, davon in aha! Neu N=35, Kompass neu $\mathrm{N}=42$, Meine Deutschtour $\mathrm{N}=67$, Magnet $\mathrm{N}=48$, Beste Freunde $\mathrm{N}=65$ (vgl. Jarząbek 2016c). 
chend benannt und mit den Angaben zur Band- und Seitennummer versehen, z.B. Beste Freunde, Sachertorte [1/60].

Die Tabelle 2 zeigt Abbildungen, die sich auf das Alltagsleben in den deutschsprachigen Ländern beziehen. Die Bilder aus A und $\mathrm{CH}$ treten in annähernd gleich hoher Zahl in den Lehrwerken auf, während Liechtensteins Alltagskontext nur durch zwei Illustrationen vertreten wird. Die meisten Bilder präsentieren typisches Essen für das jeweilige Land.

Tab. 2. Bilder mit dem Fokus auf das Alltagsleben in A, CH, L

\begin{tabular}{|c|c|c|c|}
\hline \multirow{2}{*}{ Lehrwerk } & \multicolumn{3}{|c|}{ Alltagsleben } \\
\hline & A: Bildinhalt & CH: Bildinhalt & L: Bildinhalt \\
\hline $\begin{array}{c}\text { aha! Neu } \\
\text { (Band } 1,2,3)\end{array}$ & {$[\mathrm{N}=0]$} & {$[\mathrm{N}=0]$} & {$[\mathrm{N}=0]$} \\
\hline $\begin{array}{l}\text { Beste Freunde } \\
\text { (Band } 1,2,3)\end{array}$ & $\begin{array}{c}{[\mathrm{N}=4]} \\
\text { Mozartkugel }[1 / 24] \\
\text { Sachertorte }[1 / 60] \\
\text { Mozartkugel }[3 / 55] \\
\text { Jugendhaus Salzburg } \\
{[3 / 53]}\end{array}$ & $\begin{array}{c}{[\mathrm{N}=4]} \\
\text { Lebkuchen aus Bern } \\
{[1 / 60]} \\
\text { Glacier-Express } \\
{[2 / 69]} \\
\text { Berghütte }[2 / 69] \\
\text { Käseproduktion } \\
{[2 / 69]}\end{array}$ & $\begin{array}{c}{[\mathrm{N}=1]} \\
\text { Spätzle [1/60] }\end{array}$ \\
\hline $\begin{array}{l}\text { Meine Deutschtour } \\
\quad \text { (Band } 1,2,3)\end{array}$ & $\begin{array}{c}{[\mathrm{N}=5]} \\
\text { Wiener Schnitzel } \\
{[1 / 106]} \\
\text { Wiener Schnitzel } \\
{[1 / 112]} \\
\text { Sachertorte }[1 / 112] \\
\text { Apfelstrudel }[1 / 112] \\
\text { Mozartkugel }[1 / 113]\end{array}$ & $\begin{array}{c}{[\mathrm{N}=3]} \\
\text { Rösti [1/112] } \\
\text { Fondue [1/112] } \\
\text { Jungfrau-Bahn [2/23] }\end{array}$ & $\begin{array}{c}{[\mathrm{N}=1]} \\
\text { Autokennzeichen FL } \\
{[1 / 45]}\end{array}$ \\
\hline $\begin{array}{l}\text { Kompass neu } \\
\text { (Band } 1,2,3)\end{array}$ & $\begin{array}{c}{[\mathrm{N}=1]} \\
\text { Murmeltier [3/28] }\end{array}$ & $\begin{array}{c}{[\mathrm{N}=1]} \\
\text { Stundenplan in einer } \\
\text { Schule in Gstaad } \\
{[1 / 79]}\end{array}$ & {$[\mathrm{N}=0]$} \\
\hline $\begin{array}{c}\text { Magnet } \\
\text { (Band 1, 2, 3, 4) }\end{array}$ & $\begin{array}{c}{[\mathrm{N}=1]} \\
\text { Sommercamp in Tirol } \\
{[4 / 12]}\end{array}$ & {$[\mathrm{N}=0]$} & {$[\mathrm{N}=0]$} \\
\hline Bilderzahl & 11 & 8 & 2 \\
\hline
\end{tabular}

Tabelle 3 dokumentiert das Bildmaterial aus dem Bereich Städte/Regionen/ Geografie. Es ist auffällig, dass die Abbildungen der Länder A und CH fast genauso oft in den untersuchten Lehrwerken zu finden sind. Ein häufiges Inhaltsmotiv stellen dabei Landkarten mit den deutschsprachigen Ländern bzw. ihren Regionen und Stadtpanoramas von größeren Städten wie Innsbruck, Salzburg, Bern und Zürich sowie der Hauptstadt Liechtensteins Vaduz dar. Auch die Alpenland- 
schaft und das Matterhorn gelten in den Lehrwerken als Wahrzeichen einzelner DACHL-Länder.

Tab. 3. Bilder mit dem Fokus auf Städte/Regionen/Geografie A, CH, L

\begin{tabular}{|c|c|c|c|}
\hline \multirow{2}{*}{ Lehrwerk } & \multicolumn{3}{|c|}{ Städte/Regionen/Geografie } \\
\hline & A: Bildinhalt & CH: Bildinhalt & L: Bildinhalt \\
\hline $\begin{array}{c}\text { aha! Neu } \\
\text { (Band 1, 2, 3) }\end{array}$ & $\begin{array}{c}{[\mathrm{N}=1]} \\
\text { Innsbruck }[2 \mathrm{a} / 53]\end{array}$ & {$[\mathrm{N}=0]$} & {$[\mathrm{N}=0]$} \\
\hline $\begin{array}{l}\text { Beste Freunde } \\
\text { (Band } 1,2,3)\end{array}$ & $\begin{array}{c}{[\mathrm{N}=9]} \\
\text { Landkarte } \\
{[1 / 2 \text { Umschlag] }} \\
\text { Landkarte } \\
\text { [2/2Umschlag] } \\
\text { Landkarte } \\
\text { [3/2Umschlag] } \\
\text { Innsbruck, } \\
\text { Stadtpanorama } \\
\text { [2/68] } \\
\text { Innsbruck, Lage auf } \\
\text { der Landkarte [2/68] } \\
\text { Salzburg, } \\
\text { Stadtpanorama } \\
\text { [3:49] } \\
\text { Salzburg, } \\
\text { Kapitelplatz [3/55] } \\
\text { Salzburg, Flughafen } \\
\text { [3/55] } \\
\text { Salzburg, } \\
\text { Mozarteum [3/55] }\end{array}$ & $\begin{array}{c}{[\mathrm{N}=8]} \\
\text { Landkarte } \\
{[1 / 2 \text { Umschlag] }} \\
\text { Landkarte } \\
\text { [2/2Umschlag] } \\
\text { Landkarte } \\
\text { [3/2Umschlag] } \\
\text { Matterhorn [1/24] } \\
\text { Landkarte von } \\
\text { Sprachgebieten } \\
\text { [2/69] } \\
\text { Bern, Kramgasse } \\
{[2 / 69]} \\
\text { Zürichsee [2/69], } \\
\text { Bodenseewanderweg } \\
\text { in Rorschach [2/69], }\end{array}$ & $\begin{array}{c} \\
\text { Landkarte } \\
\text { [N=3] } \\
\text { [1/2Umschlag] } \\
\text { Landkarte } \\
\text { [2/2Umschlag] } \\
\text { Landkarte } \\
\text { [3/2Umschlag] }\end{array}$ \\
\hline
\end{tabular}




\begin{tabular}{|c|c|c|c|}
\hline $\begin{array}{l}\text { Meine Deutschtour } \\
\text { (Band } 1,2,3)\end{array}$ & $\begin{array}{c}\qquad \mathrm{N}=6] \\
\text { Landkarte [1/18] } \\
\text { Landkonturen [1/22] } \\
\text { Großglockner } \\
\text { Hochalpenstraße } \\
{[2 / 23]} \\
\text { Landkarte [2/26] } \\
\text { Salzburg } \\
\text { Stadtpanorama } \\
{[1 / 22]} \\
\text { Landkarte [3/22] }\end{array}$ & $\begin{array}{c}{[\mathrm{N}=6]} \\
\text { Landkarte [1/18] } \\
\text { Bern, Stadtpanorama } \\
{[1 / 19]} \\
\text { Landkonturen [1/24] } \\
\text { Zürich, } \\
\text { Stadtpanorama } \\
{[1 / 25]} \\
\text { Landkarte [2/26] } \\
\text { Landkarte [3/22] }\end{array}$ & $\begin{array}{c}{[\mathrm{N}=6]} \\
\text { Landkarte [1/18] } \\
\text { Vaduz, } \\
\text { Stadtpanaroma mit } \\
\text { Alpen [1/19] } \\
\text { Alpenlandschaft um } \\
\text { Vaduz [1/26] } \\
\text { Vaduz [1/26] } \\
\text { Landkarte [2/26] } \\
\text { Landkarte [3/22] }\end{array}$ \\
\hline $\begin{array}{l}\text { Kompass neu } \\
\text { (Band } 1,2,3)\end{array}$ & $\begin{array}{c}{[\mathrm{N}=11]} \\
\text { Europakarte [1/2 } \\
\text { Umschlag] } \\
\text { Landkarte mit } \\
\text { Salzburg [1/14] } \\
\text { Landkarte mit Linz } \\
\text { [1/15] } \\
\text { Landkarte mit } \\
\text { A-Ausschnitt [2/2 } \\
\text { Umschlag] } \\
\text { Landkarte [2/6] } \\
\\
\text { Landkarte mit den } \\
\text { Bundesländern [2/7] } \\
\\
\text { Ringstraße [2/126] } \\
\text { Stadtplan von Wien } \\
\text { [2/127] } \\
\text { Weltkarte [3/2 } \\
\text { Umschlag] } \\
\text { Naturpark } \\
\text { Vivarium [3/29] } \\
\text { Narzissenfelder in } \\
\text { Steiermark [3/29] }\end{array}$ & $\begin{array}{c}{[\mathrm{N}=9]} \\
\text { Europakarte [1/2 } \\
\text { Umschlag] } \\
\text { Landkarte mit CH- } \\
\text { Ausschnitt [2/2 } \\
\text { Umschlag] } \\
\text { Landkarte [2/6] } \\
\text { Landkarte mit } \\
\text { Kantonen [2/7] } \\
\text { Zürich, } \\
\text { Stadtpanorama [2/7] } \\
\text { Bern, Stadtpanorama } \\
\text { [2/7] } \\
\text { Weltkarte [3/2 } \\
\text { Umschlag] } \\
\text { Zermatt-Dorf [3/28] } \\
\text { Matterhorn [3/28] }\end{array}$ & $\begin{array}{c}\quad[\mathrm{N}=2] \\
\text { Europakarte }[1 / 2 \\
\text { Umschlag] } \\
\text { Weltkarte }[3 / 2 \\
\text { Umschlag] }\end{array}$ \\
\hline
\end{tabular}




\begin{tabular}{|c|c|c|c|}
\hline $\begin{array}{c}\text { Magnet } \\
\text { (Band 1, 2, 3, 4) }\end{array}$ & $\begin{array}{c}\text { [N=10] } \\
\text { Landkarte } \\
\text { [1/2Umschlag] } \\
\text { Landkarte } \\
\text { [2/2Umschlag] } \\
\text { Landkarte } \\
\text { [3/2Umschlag] } \\
\text { polit. Landkarte } \\
\text { [1/3Umschlag] } \\
\text { polit. Landkarte } \\
\text { [2/3Umschlag] } \\
\text { polit. Landkarte } \\
\text { [3/3Umschlag] } \\
\text { Landkarte [1/19] } \\
\text { Landkarte [1/21] } \\
\text { Innsbruck, } \\
\text { Alpenlandschaft } \\
\text { [1/19] } \\
\text { Salzburg, } \\
\text { Stadtpanorama } \\
\text { [1/19] }\end{array}$ & $\begin{array}{c}{[\mathrm{N}=9]} \\
\text { Landkarte } \\
{[1 / 2 \text { Umschlag] }} \\
\text { Landkarte } \\
\text { [2/2Umschlag] } \\
\text { Landkarte } \\
\text { [3/2Umschlag] } \\
\text { polit. Landkarte } \\
\text { [1/3Umschlag] } \\
\text { polit. Landkarte } \\
\text { [2/3Umschlag] } \\
\text { polit. Landkarte } \\
\text { [3/3Umschlag] } \\
\text { Bern, Stadtpanorama } \\
\text { [1/19] Landkarte } \\
\text { [1/19] } \\
\text { Landkarte [1/21] }\end{array}$ & $\begin{array}{l}\qquad \mathrm{N}=3] \\
\text { polit. Landkarte } \\
\text { [1/3Umschlag] } \\
\text { polit. Landkarte } \\
\text { [2/3Umschlag] } \\
\text { polit. Landkarte } \\
\text { [3/3Umschlag] }\end{array}$ \\
\hline Bilderzahl & 37 & 32 & 14 \\
\hline
\end{tabular}

Das häufigste Bildmotiv aus dem Bereich Politik/Soziales/Geschichte sind die Nationalflaggen der deutschsprachigen Länder und sie sind in jedem evaluierten DaF-Lehrwerk zu finden (vgl. Tab. 4).

Tab. 4. Bilder mit dem Fokus auf Politik/Soziales/Geschichte A, CH, L

\begin{tabular}{|c|c|c|c|}
\hline \multirow{2}{*}{ Lehrwerk } & \multicolumn{3}{|c|}{ Politik/Soziales/Geschichte } \\
\cline { 2 - 4 } & A: Bildinhalt & $\mathbf{C H}$ : Bildinhalt & L: Bildinhalt \\
\hline $\begin{array}{c}\text { aha! Neu } \\
\text { Band } 1,2,3)\end{array}$ & $\begin{array}{c}{[\mathrm{N}=1]} \\
\text { Nationalfahne } \\
{[2 \mathrm{a} / 17]}\end{array}$ & $\begin{array}{c}\text { Nationalfahne } \\
{[2 \mathrm{a} / 17]}\end{array}$ & {$[\mathrm{N}=0]$} \\
\hline $\begin{array}{c}\text { Beste Freunde } \\
\text { (Band } 1,2,3)\end{array}$ & {$[\mathrm{N}=0]$} & $\begin{array}{c}{[\mathrm{N}=1]} \\
\text { Nationalfahne }[1 / 22]\end{array}$ & {$[\mathrm{N}=0]$} \\
\hline
\end{tabular}




\begin{tabular}{|c|c|c|c|}
\hline $\begin{array}{l}\text { Meine Deutschtour } \\
\quad(\text { Band } 1,2,3)\end{array}$ & $\begin{array}{l}\qquad \mathrm{N}=4] \\
\text { Nationalfahne }[1 / 22] \\
\text { Nationalfahne }[1 / 35] \\
\text { Nationalfahne und } \\
\text { Landkonturen }[1 / 45] \\
\text { Nationalfahne }[1 / 55]\end{array}$ & $\begin{array}{c}{[\mathrm{N}=2]} \\
\text { Nationalfahne }[1 / 24] \\
\text { Nationalfahne und } \\
\text { Landkonturen }[1 / 45]\end{array}$ & $\begin{array}{c}{[\mathrm{N}=3]} \\
\text { Nationalfahne }[1 / 26] \\
\text { Grenze zwischen L } \\
\text { und } \mathrm{CH} \text { am Rhein } \\
{[1 / 26]} \\
\text { Nationalfahne und } \\
\text { Landkonturen }[1 / 45]\end{array}$ \\
\hline $\begin{array}{l}\text { Kompass neu } \\
\text { (Band 1, 2, 3) }\end{array}$ & $\begin{array}{c}{[\mathrm{N}=1]} \\
\text { Nationalfahne }[1 / 7]\end{array}$ & $\begin{array}{c}\qquad \mathrm{N}=2] \\
\text { Nationalfahne [1/7] } \\
\text { Nationalfahne [1/83] }\end{array}$ & $\begin{array}{c}{[\mathrm{N}=1]} \\
\text { Nationalfahne [1/7] }\end{array}$ \\
\hline $\begin{array}{c}\text { Magnet } \\
\text { (Band } 1,2,3,4)\end{array}$ & $\begin{array}{c}{[\mathrm{N}=1]} \\
\text { Nationalfahne [1/33] }\end{array}$ & $\begin{array}{c}{[\mathrm{N}=1]} \\
\text { Nationalfahne }[1 / 33]\end{array}$ & $\begin{array}{l}{[\mathrm{N}=1]} \\
\text { Nationalfahne [1/33] }\end{array}$ \\
\hline Bilderzahl & 7 & 7 & 5 \\
\hline
\end{tabular}

Nur die Lehrwerke Beste Freunde und Kompass neu widmen dem visuellen Material, das Sitten und Bräuche in A und CH thematisiert, einen Platz (vgl. Tab. 5). Dabei dominieren Weihnachts-, Fastnachts- und Karnevalmotive.

Tab. 5. Bilder mit dem Fokus auf Sitten/Bräuche in A, CH, L

\begin{tabular}{|c|c|c|c|}
\hline \multirow{2}{*}{ Lehrwerk } & \multicolumn{3}{|c|}{ Sitten/Bräuche } \\
\hline & A: Bildinhalt & CH: Bildinhalt & L: Bildinhalt \\
\hline $\begin{array}{c}\text { aha! Neu } \\
\text { (Band } 1,2,3)\end{array}$ & {$[\mathrm{N}=0]$} & {$[\mathrm{N}=0]$} & {$[\mathrm{N}=0]$} \\
\hline $\begin{array}{c}\text { Beste Freunde } \\
\text { (Band } 1,2,3)\end{array}$ & $\begin{array}{c}{[\mathrm{N}=3]} \\
\text { Weihnachtskekse } \\
{[3 / 20]} \\
\text { Weihnachtsessen } \\
{[3 / 20]} \\
\text { Vanillekipferl [3/20] }\end{array}$ & $\begin{array}{c}{[\mathrm{N}=5]} \\
\text { Alphorn-Spieler in } \\
\text { Leventina [2/69] } \\
\text { Basel, Fastnachtzug } \\
{[3 / 20]} \\
\text { Basel, Maskenzug } \\
{[3 / 20]} \\
\text { Fastnachtsessen, } \\
\text { Mehlsuppe [3/20] } \\
\text { Fastnachtsessen, } \\
\text { Käsewähe [3/20] }\end{array}$ & {$[\mathrm{N}=0]$} \\
\hline $\begin{array}{l}\text { Meine Deutschtour } \\
\quad(\text { Band } 1,2,3)\end{array}$ & {$[\mathrm{N}=0]$} & {$[\mathrm{N}=0]$} & {$[\mathrm{N}=0]$} \\
\hline
\end{tabular}




\begin{tabular}{|c|c|c|c|}
\hline $\begin{array}{l}\text { Kompass neu } \\
\text { (Band } 1,2,3)\end{array}$ & $\begin{array}{c}\quad[\mathrm{N}=5] \\
\text { Tracht: Dirndl [3/29] } \\
\text { Volkstanz in Aus- } \\
\text { seerland [3/29] } \\
\text { Karneval in Bad Alt- } \\
\text { aussee Steiermark, } \\
\text { Trommelweiber } \\
{[3 / 73]} \\
\text { Karneval in Bad Alt- } \\
\text { aussee Steiermark, } \\
\text { Flinserl [3/73] } \\
\text { Karneval in Bad Alt- } \\
\text { aussee Steiermark, } \\
\text { Pless [3/73] }\end{array}$ & $\begin{array}{l}\qquad \mathrm{N}=2] \\
\text { Fastnacht, Trommler } \\
\text { und Piccolospieler } \\
\text { mit Laternen [3/72] } \\
\text { Fastnacht, Guggen- } \\
\text { musiker [3/72] }\end{array}$ & {$[\mathrm{N}=0]$} \\
\hline $\begin{array}{c}\text { Magnet } \\
\text { (Band } 1,2,3,4)\end{array}$ & {$[\mathrm{N}=0]$} & {$[\mathrm{N}=0]$} & {$[\mathrm{N}=0]$} \\
\hline Bilderzahl & 8 & 7 & $\mathbf{0}$ \\
\hline
\end{tabular}

Persönlichkeiten, die mit Österreich verbunden sind, kann man in fast allen Lehrwerken identifizieren, während Personen, deren Leben und/oder Werk auf CH oder L zu beziehen wäre, nicht präsent sind (vgl. Tab. 6).

Tab. 6. Bilder mit dem Fokus auf Persönlichkeiten in A, CH, L

\begin{tabular}{|c|c|c|c|}
\hline \multirow{2}{*}{ Lehrwerk } & \multicolumn{3}{|c|}{ Persönlichkeiten } \\
\hline & A: Bildinhalt & CH: Bildinhalt & L: Bildinhalt \\
\hline $\begin{array}{c}\text { aha! Neu } \\
\text { (Band 1, 2, 3) }\end{array}$ & $\begin{array}{c}{[\mathrm{N}=1]} \\
\text { Arnold Schwarze- } \\
\text { negger }[3 \mathrm{a} / 39]\end{array}$ & {$[\mathrm{N}=0]$} & {$[\mathrm{N}=0]$} \\
\hline $\begin{array}{l}\text { Beste Freunde } \\
\text { (Band } 1,2,3 \text { ) }\end{array}$ & $\begin{array}{c}{[\mathrm{N}=2]} \\
\text { Wolfgang Amadeus } \\
\text { Mozart [3/56] } \\
\text { Maria Anna Mozart } \\
(, \text { Nannerl“") }[3 / 56]\end{array}$ & {$[\mathrm{N}=0]$} & {$[\mathrm{N}=0]$} \\
\hline $\begin{array}{c}\text { Meine Deutschtour } \\
(\text { Band } 1,2,3)\end{array}$ & {$[\mathrm{N}=0]$} & {$[\mathrm{N}=0]$} & {$[\mathrm{N}=0]$} \\
\hline $\begin{array}{l}\text { Kompass neu } \\
\text { (Band } 1,2,3)\end{array}$ & $\begin{array}{c}\quad[\mathrm{N}=2] \\
\text { Arnold Schwarze- } \\
\text { negger [3/7] } \\
\text { Wolfgang Amadeus } \\
\text { Mozart [3/117] }\end{array}$ & {$[\mathrm{N}=0]$} & {$[\mathrm{N}=0]$} \\
\hline
\end{tabular}




\begin{tabular}{|c|c|c|c|}
\hline $\begin{array}{c}\text { Magnet } \\
\text { (Band 1,2,3,4) }\end{array}$ & $\begin{array}{c}{[\mathrm{N}=3]} \\
\text { Kaiserin Elisabeth } \\
\text { (Sisi) }[4 / 40] \\
\text { Kaiser Franz Joseph } \\
{[4 / 40]} \\
\text { Kaiserin Elisabeth } \\
\text { (Sisi) }[4 / 41]\end{array}$ & & \\
& $\mathbf{8}$ & & \\
\hline Bilderzahl & & $\mathbf{0}=0]$ & \\
\hline
\end{tabular}

Die zweitgrößte Bildergruppe wird der Kategorie Kultur/Architektur/Sehenswürdigkeiten zugeordnet. Hier werden sowohl Kunstgemälde und -denkmäler als auch mehrere architektonische Sehenswürdigkeiten oder auch Umschlagseiten der österreichischen und Schweizer Zeitungen sowie Zeitschriften verortet.

Tab. 7. Bilder mit dem Fokus auf Kultur/Architektur/Sehenswürdigkeiten in A, CH, L

\begin{tabular}{|c|c|c|c|}
\hline \multirow{2}{*}{ Lehrwerk } & \multicolumn{3}{|c|}{ Kultur/Architektur/Sehenswürdigkeiten } \\
\hline & A: Bildinhalt & CH: Bildinhalt & L: Bildinhalt \\
\hline $\begin{array}{c}\text { aha! Neu } \\
\text { (Band 1, 2, 3) }\end{array}$ & $\begin{array}{c}{[\mathrm{N}=2]} \\
\text { Gemälde von Oskar } \\
\text { Kokoschka [3b/12] } \\
\text { Burgtheater in Wien } \\
{[3 \mathrm{~b} / 25]}\end{array}$ & {$[\mathrm{N}=0]$} & {$[\mathrm{N}=0]$} \\
\hline $\begin{array}{l}\text { Beste Freunde } \\
\text { (Band } 1,2,3)\end{array}$ & $\begin{array}{c}\quad[\mathrm{N}=3] \\
\text { Riesenrad auf dem } \\
\text { Wiener Prater [1/22] } \\
\text { Stephansdom [2/38] } \\
\text { Salzburg, Mozarts } \\
\text { Geburtshaus }[3 / 56]\end{array}$ & {$[\mathrm{N}=0]$} & $\begin{array}{c}{[\mathrm{N}=1]} \\
\text { Schloss Vaduz } \\
{[1 / 24]}\end{array}$ \\
\hline $\begin{array}{l}\text { Meine Deutschtour } \\
\quad(\text { Band } 1,2,3)\end{array}$ & $\begin{array}{c}{[\mathrm{N}=8]} \\
\text { „Hundertwasserschule“ } \\
\text { in Wien }[1 / 77] \\
\text { Johann-Strauss- } \\
\text { Denkmal in Wien } \\
{[1 / 19]} \\
\text { Innsbruck Skischanze } \\
{[1 / 22]} \\
\text { Schloss Schönbrunn } \\
{[1 / 113]} \\
\text { Stephansdom [1/113] } \\
\text { Hundertwasserhaus } \\
{[1 / 113]} \\
\text { Riesenrad im Wiener } \\
\text { Prater [1/113] } \\
\text { Naturhistorisches } \\
\text { Museum in Wien } \\
{[3 / 32]}\end{array}$ & $\begin{array}{c}\text { } \\
\text { [N=4] } \\
\text { Genf. ONZ-Sitz } \\
{[1 / 25]} \\
\text { Lausanne, Denkmal } \\
\text { vor dem IOK }[1 / 25] \\
\text { Basel, Rotes Rathaus } \\
{[1 / 25]} \\
\text { Verkehrshaus in } \\
\text { Luzern }[3 / 32]\end{array}$ & $\begin{array}{c}{[\mathrm{N}=1]} \\
\text { Schloss Vaduz } \\
{[2 / 23]}\end{array}$ \\
\hline
\end{tabular}




\begin{tabular}{|c|c|c|c|}
\hline $\begin{array}{l}\text { Kompass neu } \\
\text { (Band } 1,2,3)\end{array}$ & $\begin{array}{c}{[\mathrm{N}=10]} \\
\text { Johann-Strauss- } \\
\text { Denkmal in Wien [1/8] } \\
\text { Schloss Schönbrunn } \\
{[2 / 7]} \\
\text { Hundertwasserhaus } \\
{[2 / 83]} \\
\text { Wien, Fiaker am } \\
\text { Schloss Schönbrunn } \\
{[2 / 126]} \\
\text { Stephansdom [2/126] } \\
\text { KunstHausWien } \\
{[2 / 126]} \\
\text { Riesenrad auf dem } \\
\text { Wiener Prater [2/126] } \\
\text { Riesenrad auf dem } \\
\text { Wiener Prater [2/127] } \\
\text { Zeitungen in A (Die } \\
\text { Presse, Kurier, Der } \\
\text { Standard) [3/110] } \\
\text { „Kuss“ Klimt [3/115] }\end{array}$ & $\begin{array}{c}{[\mathrm{N}=5]} \\
\text { Bern, } \\
\text { Arkadenpromenade } \\
{[1 / 8] \text { Bern, }} \\
\text { Arkadenpromenade } \\
{[1 / 28]} \\
\text { Zentrum Paul Klee in } \\
\text { Bern [2/82] } \\
\text { Presse in CH (.ch, } \\
\text { Neue } \\
\text { Züricher Zeitung, } \\
\text { Spick Klugscheisser. } \\
\text { ch) [3/110] } \\
\text { Gemälde von Paul } \\
\text { Klee [3/115] }\end{array}$ & {$[\mathrm{N}=0]$} \\
\hline $\begin{array}{c}\text { Magnet } \\
\text { (Band 1, 2, 3, 4) }\end{array}$ & $\begin{array}{c}{[\mathrm{N}=3]} \\
\text { Hofburg }[1 / 29] \\
\text { Schloss Schönbrunn } \\
{[2 / 7]} \\
\text { Schloss Schönbrunn } \\
{[4 / 12]}\end{array}$ & $\begin{array}{l}\qquad \mathrm{N}=1] \\
\text { Züricher Dom [1/19] }\end{array}$ & {$[\mathrm{N}=0]$} \\
\hline Bilderzahl & 26 & 10 & 2 \\
\hline
\end{tabular}

\section{Diskussion}

An dieser Stelle werden die Ergebnisse der Bildanalyse diskutiert. Im Deutschlehrbuch Kompass neu befinden sich die meisten Fotos mit landeskundlichen Inhalten, was Tabelle 1 dokumentiert. Einige Bilder in diesem Lehrwerk werden aber gar nicht unterschrieben (vgl. z.B. Kompass neu 2, S. 7). Die Lernenden können aus dem Kontext der gesamten Lehrwerkeinheit nicht immer eindeutig erschließen, welches Schloss bzw. Stadtpanorama auf einem Foto dargestellt ist. Das Foto hat dann lediglich eine dekorative Funktion und wenig didaktischen Nutzen.

Das Lehrwerk Magnet präsentiert auf der zweiten und vorletzten Umschlagseite jedes Bandes jeweils eine physische und politische Landkarte mit dem Titel „Die deutschsprachigen Länder“. Es fällt aber auf, dass auf der physischen Landkarte nur Konturen des Fürstentums Lichtenstein markiert, jedoch weder der Name des Landes noch der Hauptstadt vermerkt sind. Dies fällt auch deshalb besonders ins Auge, da im Falle der anderen deutschsprachigen Länder diese An- 
gaben gemacht werden. Außerdem ist die unterschiedliche grafische Markierung der Namen der Hauptstädte zu bemerken. Während Berlin visuell sehr auffällig markiert ist, lässt die grafische Kennzeichnung der anderen Hauptstädte zu wünschen übrig. Darüber hinaus werden in Magnet 1 (S. 19, 21) die Grenzen der drei deutschsprachigen Länder bis auf Liechtenstein bunt konturiert.

Es muss angemerkt werden, dass es in den evaluierten DaF-Lehrwerken auch Texte über andere Aspekte der deutschsprachigen Länder gibt, als nur diese, die die angenommenen Bildkategorien umfassen. Die Texte werden jedoch nicht immer mit passenden Bildern versehen, wie z.B. ein Textausschnitt über Reinhold Messner in Magnet 4 (45). Somit wird kein anderes Land, in dem es deutschsprachige Regionen gibt, wie z.B. Luxemburg, Belgien oder eine Region wie Südtirol visuell dargestellt.

Die vorliegende Lehrwerkanalyse zeigt, dass das ikonografische Material in den untersuchten DaF-Lehrwerken größtenteils aus Fotos besteht. Es wird aber mit wenigen Zeichnungen (z.B. Länderkonturen, Autoschildern, Nationalfahnen), Reproduktionen von Gemälden, Landkarten oder Ausschnitten von Stadtplänen ergänzt. Die meisten Bilder befinden sich im Lehrwerk Kompass neu [N=53], gefolgt von Meine Deutschtour [49], Beste Freunde [43] und Magnet [N=33]. Das Lehrwerk aha! Neu beinhaltet insgesamt nur sechs Bilder der anderen deutschsprachigen Länder als Deutschland. ${ }^{10}$ Der quantitative Unterschied zu den anderen evaluierten DaF-Lehrwerken ist somit signifikant.

Es ist auffällig, dass das Bild Liechtensteins in diesen DaF-Lehrwerken unterrepräsentiert und fast nur auf das Schloss Vaduz bzw. auf das Vaduzer Stadtpanorama oder aber die Nationalfahne reduziert wird. Die Illustrationen zu Österreich und zur Schweiz stehen ebenfalls in keinem Gleichgewicht, denn Österreich wird um ein Drittel häufiger visuell dargestellt als die Schweiz.

Schauen wir uns die Bildinhalte näher an, wird der alltägliche Kontext in den drei Ländern vor allem durch typische Nationalgerichte widergespiegelt. Die österreichischen und schweizerischen Städte, vorwiegend Wien, Innsbruck, Salzburg, Bern und Zürich sowie die Alpenimpressionen dieser Länder, visualisiert durch das Matterhorn, Zermatt oder eine unbestimmte Alpenlandschaft, werden als Attraktionen wie auf Postkarten oder touristischen Werbeflyern präsentiert. Lobenswert ist ein reichliches Angebot an Landkarten in jedem Lehrwerk, was die räumliche Bestimmung der Länder, der Städte und der Regionen in Europa (im Falle von Kompass neu auch in der Welt) den Lernenden wesentlich erleichtern kann. Im Lehrwerk aha! Neu fehlen allerdings die Landkarten. Nur in Beste Freunde 2 (S. 69) wird die einzige Schweizer Landkarte mit markierten Sprachgebieten dieses viersprachigen Landes gezeigt. Die Bilder mit geografischem Kontext überwiegen im ikonografischen Material der evaluierten Lehrwerke.

10 Das Deutschlandbild wird im Lehrwerk aha! Neu mit 35 Abbildungen widergeben (vgl. Jarząbek 2016c). 
Dem Bereich Politik können die Nationalfahnen der deutschsprachigen Länder zugordnet werden und diese sind fast in jedem Buch vorhanden. Die Illustrationen von Sitten und Bräuchen oder (welt)bekannten Persönlichkeiten aus ACHL-Ländern gehören nicht zu häufigen Bildmotiven in den Lehrwerken. Die Abbildungen von Kaiser Franz Joseph und seiner Ehefrau Elisabeth sowie Arnold Schwarzenegger und Wolfgang Amadeus Mozart stehen für Geschichte und Kultur Österreichs, und zwar nur in drei der untersuchten Lehrwerke.

Die Fotos österreichischer Sehenswürdigkeiten dominieren in der Bildkategorie Kultur/Architektur/Sehenswürdigkeiten, und hier insbesondere das Riesenrad im Wiener Prater, der Stephansdom und das Schloss Schönbrunn. Auch das Schloss Vaduz ist als Wahrzeichen Liechtensteins in zwei Lehrwerken vorhanden. Die diversen Bilder aus der Schweiz, die in den Lehrwerken zu finden sind, lassen darüber hinaus kein Kultur- bzw. Architekturobjekt als Symbol des Landes klar erkennen.

\section{Fazit}

Die durchgeführte Analyse des visuellen Materials spricht von dem Ist-Stand bei fünf von zwölf DaF-Lehrwerksreihen, die in polnischen Gymnasien im DaF-Unterricht gebraucht werden dürfen und dort am häufigsten eingesetzt werden.

Maijala u.a. haben eine ähnliche Bildanalyse in finnischen und niederländischen DaF-Lehrwerken durchgeführt (vgl. Maijala/Tammenga-Helmantel/Donker 2016) und eine Überzahl der Bilder aus Deutschland festgestellt, während die Anzahl von Bildern aus Österreich und der Schweiz vergleichbar niedrig ist. Liechtenstein oder andere deutschsprachige Halbzentren treten in finnischen DaF-Lehrwerken überhaupt nicht auf. Wie im polnischen Falle gleichen die Fotomotive den bekannten Lehrwerkstereotypen, wie die Nationalfahne, die Alpen, Mozartkugeln und Käse, touristische Attraktionen, Traditionen und Feste. Dies kann eine Festlegung von Funk (2004: 41) bestätigen, dass sich die DaF-Lehrwerke nicht nur deutscher Verlage, sondern auch regionale DaF-Lehrbücher, wie die hier untersuchten polnischen Lehrwerke, wesentlich angeglichen haben. Dafür spricht auch die Tatsache, dass überhaupt die meisten Bilder der untersuchten DaF-Lehrwerke Menschen darstellen, die den Lehrwerktexten ihr Gesicht und damit die gewünschte Authentizität verleihen sollten. Im Falle polnischer Deutschlehrwerke ist ebenso auffällig, dass gerade diese Fotos immer öfters von globalen Bildanbietern wie beispielsweise Shutterstock stammen. Damit wird ihre Authentizität jedoch in Frage gestellt.

Darüber hinaus stellt sich das Bild der deutschsprachigen Länder in den polnischen DaF-Lehrwerken, auch wenn das vielleicht nur für die Lehrbücher im polnischen Gymnasium, d.h. für die Klassen 7 bis 9, zutrifft, als einwandfrei und touristisch verlockend dar. Polnische DaF-Lerner, meistens Beginner, können dem 
ikonografischen Material vier fast ideale Länder entnehmen. Für ihre Wahrzeichen stehen vor allem bekannte Städte, repräsentative Bauten und wunderschöne (Alpen)Landschaften, jedoch nur wenige Persönlichkeiten wie Wolfgang Amadeus Mozart oder Arnold Schwarzenegger. Typische Gerichte sollten für den Alltag charakteristisch sein, jedoch scheint dieser Alltag, nach dem Bildmaterial zu schließen, gar nicht multikulturell zu sein.

Da es keine Gegenüberstellungen von kontrastiven Bildern gibt, die kontroverse oder sogar provokative Inhalte darstellen, müssen sich die Lerner nicht kritisch mit der Realität auseinandersetzen. Die so konzipierte Repräsentativität der Bilder kann auch meiner Meinung nach eine nachteilhafte Wirkung im Unterricht nach dem Motto „Zu viel Süßes wird zu guter Letzt sauer" haben. Ungewöhnliche Impressionen können junge Lerner viel intensiver zum handlungsorientieren Lernen und selbstständigen Recherchieren bewegen. Wenn die Bilder eindeutig sind, können sie selten interkulturelle Diskussionen auslösen (vgl. Macaire/Hosch 1999). Für mich stellen sich somit weiterführende Fragen: Wollen Deutschlehrende und vor allem -lernende sich auch mit anderen (Bild)Inhalten auseinandersetzten, die provokativ, untypisch und kontrovers sind? Welches Bildmaterial kann kritische Ansichten bei den Lernenden entwickeln? Welche Fotos können beispielsweise besser Diskussionen über (inter)kulturelle Missverständnisse im Alltagsleben einleiten? Die Antworten darauf bedürfen jedoch noch weiterer Untersuchungen.

\section{Literatur}

„ABCD-Thesen zur Rolle der Landeskunde im Deutschunterricht“. In: Fremdsprache Deutsch 1990, H. 3. S. 60-61.

Badstübner-Kizik, Camilla (2006): Fremde Sprachen - fremde Künste? Bild- und Musikkunst im Fremdsprachenunterricht. Das Fallbeispiel Deutsch als Fremdsprache in Polen. Gdańsk.

Badstübner-Kizik, Camilla (2007): Bild- und Musikkunst im Fremdsprachenunterricht. Zwischenbilanz und Handreichungen für die Praxis. Frankfurt am Main.

Breitung, Horst / Lattaro, Elisabeth (2001): Regionale Lehrwerke und Lehrmethoden. In: Helbig, Gerhard / Götze, Lutz / Henrici, Gert / Krumm, Hans-Jürgen (Hrsg.): Deutsch als Fremdsprache. Ein internationales Handbuch. 2. Halbband. Berlin/New York. S. 1041-1053.

Bucher, Hans-Jürgen / Schumacher, Peter (Hrsg.) (2012): Interaktionale Rezeptionsforschung. Theorie und Methode der Blickaufzeichnung in der Medienforschung. Wiesbaden.

Chudak, Sebastian (Hrsg.) (2013): Fremdsprachenunterricht-ominmedial? Frankfurt am Main.

Demmig, Silvia / Hägi, Sara / Schweiger, Hannes (Hrsg.) (2013): DACH-Landeskunde. Theorie Geschichte-Praxis. München.

Funk, Hermann (2004): Qualitätsmerkmale von Lehrwerken prüfen - ein Verfahrensvorschlag. In: Babilonia. B. 3. S. 41-47.

Hieronimus, Marc (Hrsg.) (2014): Visuelle Medien im DaF-Unterricht. Göttingen.

Jarząbek, Alina Dorota (2013a): Mehrsprachigkeit und ihre Widerspiegelung in den polnischen Lehrwerken für den DaF-Unterricht. In: Grucza, Franciszek / Zhu, Jianhua (Hrsg.): Akten des 
XII. Internationalen Germanistenkongresses. Publikationen der internationalen Vereinigung für Germanistik (IVG). Bd. 19. Frankfurt am Main. S. 247-254.

Jarząbek, Alina Dorota (2013b): Der Plurizentrische Ansatz und das DACH-Prinzip in polnischen DaF-Lehrwerken. In: Germanica Wratislaviensia 138. S. 173-183.

Jarząbek, Alina Dorota (2016a): Zadania stużace afektywnym celom ksztatcenia międzykulturowego $w$ podręcznikach do nauczania języka niemieckiego w gimnazjum. In: Jaroszewska, Anna Karpeta-Peć / Beata, Smuk / Maciej Wojciech / Sobańska, Joanna / Sujecka-Zając, Jolanta (Hrsg.): Wielojęzyczność i międzykulturowość na lekcji języka obcego - między teorią a praktyka nauczania. Warszawa, S. 189-202.

Jarząbek, Alina Dorota (2016b): Deutschlandbild in den DaF-Lehrwerken für polnisches Gymnasium. In: Jarząbek, Alina Dorota (Hrsg.): Anfang. Glottodidaktische Implikationen des Anfangs. Würzburg. S. 115-127.

Jarząbek, Alina Dorota (2017): Zadania rozwijające kompetencję międzykulturowa w podręcznikach do nauczania języka niemieckiego w gimnazjum. In: Prace Językoznawcze, XIX/1. S. 51-70.

Kast, Bernd / Neuner, Gerhard (Hrsg.) (1994): Zur Analyse, Begutachtung und Entwicklung von Lehrwerken. Berlin/München/Wien/Zürich/New York.

Krumm, Hans-Jürgen / Ohms-Duschenko, Maren (2001): Lehrwerkproduktion. Lehrwerkanalyse, Lehrwerkritik. In: Helbig, Gerhard/Götze, Lutz/Henrici, Gert / Krumm, Hans-Jürgen (Hrsg.): Deutsch als Fremdsprache. Ein internationales Handbuch. 2. Halbband. Berlin/New York. S. 1029-1041.

Krumm, Hans-Jürgen (2010): Lehrwerke im Deutsch als Fremd- und Zweitsprache-Unterricht. In: Krumm, Hans-Jürgen / Fandrych, Christian / Hufeisen, Britta / Riemer, Claudia (Hrsg.): Deutsch als Fremd-und Zweitsprache. Ein internationales Handbuch. Bd. 2. Berlin/New York. S. 1213-1227.

Macaire, Dominique / Hosch, Wofram (1999): Bilder in der Landeskunde. München.

Maijala, Minna / Tammenga-Helmantel, Marjon / Donker, Eva (2016): Das DACH-Konzept in finnischen und niederländischen DaF-Lehrwerken. In: Zielsprache Deutsch. H. 1. S. 3-34.

Pešková, Karolina (2014): Inhaltsanalyse der landeskundlichen Visualisierungen in DaF-Lehrwerken. In: Knecht, Peter / Matthes, Eva / Schütze, Sylvia / Aamotsbakken, Bente (Hrsg.): Methodologie und Methoden der Schulbuch- und Lehrmittelforschung. Methodology and Methods of Research on Textbooks and Educational Media. Bad Heilbrunn. S. 241-253.

Rösler, Dietmar (2010): Die Funktion von Medien im Deutsch als Fremd- und Deutsch als Zweitsprache-Unterricht. In: Krumm, Hans-Jürgen / Fandrych, Christian / Hufeisen, Britta / Riemer, Claudia (Hrsg.): Deutsch als Fremd- und Zweitsprache. Ein internationales Handbuch. Bd. 2. Berlin/New York. S. 1199-1214.

Schmidt, Reiner (2001): Lehrwerkanalyse. In: Henrici, Gert / Riemer, Claudia (Hrsg.): Einführung in die Didaktik des Unterrichts Deutsch als Fremdsprache mit Videobeispielen. Bd. II. Hohengehren. S. 367-418.

Schöttler, Tobias (2014): Was ist Bildwissenschaft? Ihre Gegenstände, Fragen und Methoden. In: Hieronimus, Marc (Hrsg.): Visuelle Medien im DaF-Unterricht. Göttingen. S. 3-24.

Schwertfeger, Inge C. (2001): Die Funktion der Medien in den Methoden des Deutsch als Fremdsprache-Unterrichts. In: Helbig, Gerhard / Götze, Lutz / Henrici, Gert / Krumm, Hans-Jürgen (Hrsg.): Deutsch als Fremdsprache. Ein internationales Handbuch. 2. Halbband. Berlin/New York. S. 1018-1028.

Sorger, Brigitte (2013): Institutions- und sprachenpolitische Aspekte des DACH-Konzepts. In: Demmig, Silvia / Hägi, Sara / Schweiger, Hannes (Hrsg.): DACH-Landeskunde. Theorie - Geschichte-Praxis. München. S. 32-48.

Spaniel-Weise, Dorothea (2014): Evaluationskriterien audio-visueller Verbundmedien für den DaF-/ DaZ-Unterricht. In: Hieronimus, Marc (Hrsg.): Visuelle Medien im DaF-Unterricht. Göttingen. S. 53-76.

Germanica Wratislaviensia 143, 2018

(C) for this edition by CNS 


\section{Internetquellen}

Duden Wörterbuch, www.duden.de/suchen/dudenonline/Bild (Zugriff am 10.07.2016)

www.idvnetz.org/dachl-online/das-dach-prinzip (Zugriff am 20.07.2016).

\section{Analysierte Lehrwerke}

Goergiakaki, Manuela / Bovermann, Monika / Graf-Riemann, Elisabeth/Suethe, Christiane/Schümann, Anja/Kozłowska, Elżbieta (2015): Beste Freunde 1. Hueber Verlag, München.

Goergiakaki, Manuela / Bovermann, Monika / Graf-Riemann, Elisabeth/Suethe, Christiane/Kołsut, Sławomira / Krajewska, Urszula (2015): Beste Freunde 2. Hueber Verlag, München.

Goergiakaki, Manuela / Bovermann, Monika / Graf-Riemann, Elisabeth / Suethe, Christiane / Schümann, Anja (2016): Beste Freunde 3. Hueber Verlag, München.

Kościelniak-Walewska, Ewa (2015): Meine Deutschtour1. Podręcznik do języka niemieckiego dla gimnazjum. Nowa Era, Warszawa.

Kościelniak-Walewska, Ewa (2015): Meine Deutschtour2. Podręcznik do języka niemieckiego dla gimnazjum. Nowa Era, Warszawa.

Kościelniak-Walewska, Ewa (2015): Meine Deutschtour3. Podręcznik do języka niemieckiego dla gimnazjum. Nowa Era, Warszawa.

Motta, Giorgio (2015): Magnet 1. Język niemiecki dla gimnazjum. Wydawnictwo „LektorKlett”, Poznań.

Motta, Giorgio (2015): Magnet 2. Język niemiecki dla gimnazjum. Wydawnictwo „LektorKlett”, Poznań.

Motta, Giorgio (2012): Magnet 3. Język niemiecki dla gimnazjum. Wydawnictwo „LektorKlett”, Poznań.

Motta, Giorgio (2015): Magnet 4. Język niemiecki dla gimnazjum. Wydawnictwo „LektorKlett”, Poznań.

Potapowicz, Anna / Tkaczyk, Krzysztof (2014): aha! Neu. Kurs podstawowy. Język niemiecki. Podręcznik do gimnazjum. 1. Wydawnictwa Szkolne i Pedagogiczne, Warszawa.

Potapowicz, Anna / Tkaczyk, Krzysztof (2015): aha! Neu. Kurs podstawowy. Język niemiecki. Podręcznik do gimnazjum. 2. Wydawnictwa Szkolne i Pedagogiczne, Warszawa.

Potapowicz, Anna / Tkaczyk, Krzysztof (2011): aha! Neu. Kurs podstawowy. Język niemiecki. Podręcznik do gimnazjum. 3. Wydawnictwa Szkolne i Pedagogiczne, Warszawa.

Reymont, Elżbieta / Sibiga, Agnieszka / Jezierska-Wiejak, Małgorzata (2015): Kompass neu 1. Podręcznik do języka niemieckiego dla gimnazjum. Wydawnictwo Szkolne PWN, Warszawa.

Reymont, Elżbieta / Sibiga, Agnieszka / Jezierska-Wiejak, Małgorzata (2015): Kompass neu 2. Podręcznik do języka niemieckiego dla gimnazjum. Wydawnictwo Szkolne PWN, Warszawa.

Reymont, Elżbieta / Sibiga, Agnieszka / Jezierska-Wiejak, Małgorzata (2013): Kompass neu 3. Podręcznik do języka niemieckiego dla gimnazjum. Wydawnictwo Szkolne PWN, Warszawa.

Germanica Wratislaviensia 143, 2018

(C) for this edition by CNS 